Check for updates

Cite this: Chem. Sci., 2018, 9, 8077

๑ All publication charges for this article have been paid for by the Royal Society of Chemistry

Received 22nd June 2018

Accepted 27th August 2018

DOI: $10.1039 / c 8 s c 02753 f$

rsc.li/chemical-science

\section{Unravelling the origins of ice nucleation on organic crystals $\uparrow$}

\author{
Gabriele C. Sosso, (D)*a Thomas F. Whale, (D) $\dot{t}^{\mathrm{b}}$ Mark A. Holden, (D) $\S^{\mathrm{bc}}$ \\ Philipp Pedevilla, ${ }^{d}$ Benjamin J. Murray (D ${ }^{b}$ and Angelos Michaelides (D) $d$
}

\begin{abstract}
Organic molecules such as steroids or amino acids form crystals that can facilitate the formation of ice arguably the most important phase transition on earth. However, the origin of the ice nucleating ability of organic crystals is still largely unknown. Here, we combine experiments and simulations to unravel the microscopic details of ice formation on cholesterol, a prototypical organic crystal widely used in cryopreservation. We find that cholesterol - which is also a substantial component of cell membranes is an ice nucleating agent more potent than many inorganic substrates, including the mineral feldspar (one of the most active ice nucleating materials in the atmosphere). Scanning electron microscopy measurements reveal a variety of morphological features on the surfaces of cholesterol crystals: this suggests that the topography of the surface is key to the broad range of ice nucleating activity observed (from -4 to $-20^{\circ} \mathrm{C}$ ). In addition, we show via molecular simulations that cholesterol crystals aid the formation of ice nuclei in a unconventional fashion. Rather than providing a template for a flat ice-like contact layer (as found in the case of many inorganic substrates), the flexibility of the cholesterol surface and its low density of hydrophilic functional groups leads to the formation of molecular cages involving both water molecules and terminal hydroxyl groups of the cholesterol surface. These cages are made of 6- and, surprisingly, 5-membered hydrogen bonded rings of water and hydroxyl groups that favour the nucleation of hexagonal as well as cubic ice (a rare occurrence). We argue that the phenomenal ice nucleating activity of steroids such as cholesterol (and potentially of many other organic crystals) is due to (i) the ability of flexible hydrophilic surfaces to form unconventional ice-templating structures and (ii) the different nucleation sites offered by the diverse topography of the crystalline surfaces. These findings clarify how exactly organic crystals promote the formation of ice, thus paving the way toward deeper understanding of ice formation in soft and biological matter - with obvious reverberations on atmospheric science and cryobiology.
\end{abstract}

\section{Introduction}

The freezing of liquid water into crystalline ice is a ubiquitous phenomenon which is part of our everyday experience ${ }^{1}$ and has countless reverberations in fields as diverse as cryobiology $y^{2-4}$ and atmospheric science. ${ }^{5,6}$ Strikingly, the overwhelming majority of ice on earth forms heterogeneously, i.e. thanks to the presence of substances, other than water itself, which facilitate the ice nucleation process. ${ }^{5,7}$ Much of what is known

\footnotetext{
${ }^{a}$ Department of Chemistry and Centre for Scientific Computing, University of Warwick, Gibbet Hill Road, Coventry CV4 7AL, UK. E-mail: g.sosso@warwick.ac.uk ${ }^{b}$ School of Earth and Environment, University of Leeds, Leeds LS2 9JT, UK 'Chemistry, University of Leeds, Leeds LS2 9JT, UK

${ }^{d}$ Thomas Young Centre, London Centre for Nanotechnology and Department of Physics and Astronomy, University College London, London WC1E 6BT, UK

$\dagger$ Electronic supplementary information (ESI) available. See DOI: $10.1039 / \mathrm{c} 8 \mathrm{sc} 02753 \mathrm{f}$

\$ School of Chemistry, University of Leeds, Leeds, LS2 9JT, UK.

$\S$ School of Physics and Astronomy, University of Leeds, Leeds, LS2 9JT, UK.
}

about heterogeneous ice nucleation has come from the study of atmospherically relevant ice nucleating agents: ${ }^{5}$ in fact, heterogeneous ice nucleation from supercooled water plays a critical role in the glaciation of mixed phase clouds, which in turn influences the climate. ${ }^{\mathbf{8} 9}$ A variety of substances are known to nucleate ice efficiently in the atmosphere, including inorganic species such as silver iodide, ${ }^{\mathbf{1 0}}$ feldspar $^{\mathbf{1 1 - 1 3}}$ as well as biological entities such as the bacterium Pseudomonas syringae ${ }^{14-16}$ or birch pollen. ${ }^{17}$

Biological ice nucleating agents also play a key role in the ever-growing field of cryobiology: in fact, the formation of ice in biological matter is the cornerstone of cryotherapy and cryopreservation, ${ }^{\mathbf{4}, 18}$ i.e. the long-term storage of frozen biological material which is essential to enable cutting-edge technologies such as regenerative medicine. ${ }^{19,20}$ A number of organic crystals have been known to facilitate ice nucleation, ${ }^{21,22}$ and molecular crystals of steroids such as cholesterol $(\mathrm{CHL})^{23}$ are used to boost the formation of ice when cryopreserving biological material. ${ }^{2,24}$ Importantly, $\mathrm{CHL}$ 
molecules also represent a major component (up to $40 \%$ ) of animal cell membranes, ${ }^{25}$ thus prompting the question of whether this steroid can play a role as ice nucleator in the context of ice formation in biological matter.

However, the microscopic details of heterogeneous ice nucleation on CHL - and indeed on the vast majority of organic and inorganic compounds alike - remain remarkably poorly understood, ${ }^{7}$ although a substantial body of experimental work has been devoted to assess the ice nucleation ability of biological matter. ${ }^{22,26-32}$ In fact, the reason why many biological ice nucleating agents display a far stronger ice nucleating activity than most inorganic materials ${ }^{5,7}$ is still largely unknown. Partly, this is because obtaining molecular-level insight into the nucleation process is still a formidable challenge for experiments, and only very recently simulations of heterogeneous ice nucleation have become feasible, ${ }^{7,13,33-40}$ largely thanks to the capabilities of the coarse grained mW water model. ${ }^{41}$ Indeed, $\mathrm{mW}$ has played a pivotal role in enabling systematic investigations of ice nucleation on e.g. carbonaceous ${ }^{42}$ or hydroxylated organic surfaces. ${ }^{43}$ However, fully atomistic water models and enhanced sampling methods are often required to take into account the subtleties of the hydrogen bond network between water and complex impurities. ${ }^{\mathbf{4 4 , 4 5}}$

In this work, we bring together experiments and simulations to take an ambitious step forward in furthering our understanding of ice formation on organic crystals. We focus on CHL, due to its relevance in cryopreservation and its role within cellular membrane, unravelling microscopic motivations for heterogeneous ice nucleation likely to be shared by many other organic crystals. We find via micro-litre droplet nucleation measurements ( $\mu$ l-NIPI) that CHL crystals display an outstanding ice nucleation ability (stronger than most inorganic ice nucleating agents), with freezing events initiating at very mild supercooling $\Delta T_{\mathrm{S}}=T_{\text {Melt }}-T=4 \mathrm{~K}$ down to $\Delta T_{\mathrm{S}}=20$ K. Scanning electron microscopy suggests that the activity of these crystals as ice nucleating agents across such a wide temperature range could be due to the diverse topography of the surface of the cholesterol crystals, which are likely to offer a variety of different nucleation sites. In order to get a molecular-level insight into the mechanism of ice formation on CHL crystals, we harness enhanced sampling simulations, focusing on the hydroxylated (001) face of cholesterol monohydrate (CHLM) - the most relevant surface (and polymorph, as discussed below) in biological scenarios. We find that CHL crystals facilitate the formation of ice in a non-conventional fashion: in contrast to what has been observed in the case of inorganic substrates such as e.g. carbonaceous particles ${ }^{\mathbf{4 2}}$ or clay minerals, ${ }^{45}$ the flexibility of the CHLM surface and the large spacing of its hydroxyl groups prevent the formation of a flat, ice-like layer of water molecules at the water-crystal interface. Instead, the hydroxyl groups participate in the formation of 5and 6-membered hydrogen bonded rings of water molecules forming peculiar molecular "cages" that provide an effective template for the nucleation of both cubic and hexagonal ice (a rare occurrence).

As a whole, our findings suggest that the formation of ice on CHL crystals originates from the ability of their flexible hydrophilic surfaces to trigger the formation of unconventional ice-templating molecular features. In addition, different nucleation sites potentially offered by the diverse topography of the crystal can further enhance the intrinsic ice nucleation potential of CHL surfaces. This insight could help to understand ice formation on a number of other organic compounds, from amino acid crystals ${ }^{\mathbf{4 6 7 4}}$ to bacterial fragments ${ }^{\mathbf{4 8 , 4 9}}$ - as they are all characterised by the presence of flexible hydrophilic surfaces displaying diverse topological features. In addition, organic crystals such as cholesterol are positioned "in between" inorganic and biological ice nucleating agents: they possess the order and the crystalline surfaces of the former, and the complexity and flexibility of the latter. This work thus paves the way to a molecular-level understanding of ice formation in biological matter, tackling a substrate (CHL crystals) that embeds unique features of very different classes of materials.

\section{Methods}

\section{$2.1 \mu \mathrm{l}$-NIPI experiments and scanning electron microscopy measurements}

The ice nucleation efficiency of CHLM was evaluated using an adapted version of the $\mu \mathrm{l}$ Nucleation by Immersed Particles Instrument ( $\mu \mathrm{l}$-NIPI) described in detail in ref. 50. To make the flat plates we dissolved $2 \mathrm{~g}$ of pure CHL (Sigma Aldrich) in approximately $30 \mathrm{ml}$ of hot ( 343 K) 95\% ethanol (SigmaAldrich). The CHL solution was then allowed to cool slowly, causing crystallisation of large (up to approx. $1 \mathrm{~cm}$ across) flat plates of CHLM. Individual plates of CHLM of around $2 \mathrm{~mm}$ diameter were then recovered by vacuum filtration on a filter membrane and placed onto a thin $(\sim 0.1 \mathrm{~mm})$ glass plate. The glass plate was then placed onto an EF600 Stirling cryocooler. A Picus Biohit electronic pipette was then used to deposit $1 \mu \mathrm{l}$ droplets of MilliQ water onto the separated CHLM plates. The EF600 cryocooler was then used to reduce the temperature of the droplets at a rate of $1 \mathrm{~K} \mathrm{~min}^{-1}$ freezing was monitored using a camera. In this way the droplet fraction frozen curve presented in Fig. 1a was built up. The data is the result of several cooling runs as only about 10 droplets could be frozen per experiment. It was important that plates were not in contact as ice clearly propagated across the CHL surface, triggering neighbouring droplets after an initial freezing events, when multiple droplets were placed on a single plate.

In order to calculate the (surface) density of the active ice nucleation sites $\left(n_{\mathrm{s}}\right.$, commonly used to compare the ice nucleating efficiency of different substances ${ }^{5}$ ) on the CHLM surface (reported in Fig. 1) we have used the following expression:

$$
\frac{n(T)}{N}=1-\exp \left[-n_{s}(T) A\right]
$$

where $n(T)$ is the number of droplets frozen at temperature $T, N$ is the total number of droplets in the experiment and $A$ is the surface area of nucleating agent per droplet. The value of $A$ for each droplet was measured using the image analysis software imageJ, customarily used to quantify particles size in biosciences. ${ }^{51}$ The resulting estimate of the mean value of $A$ is $1.82 \pm$ $0.46 \mathrm{~mm}^{2}$. 


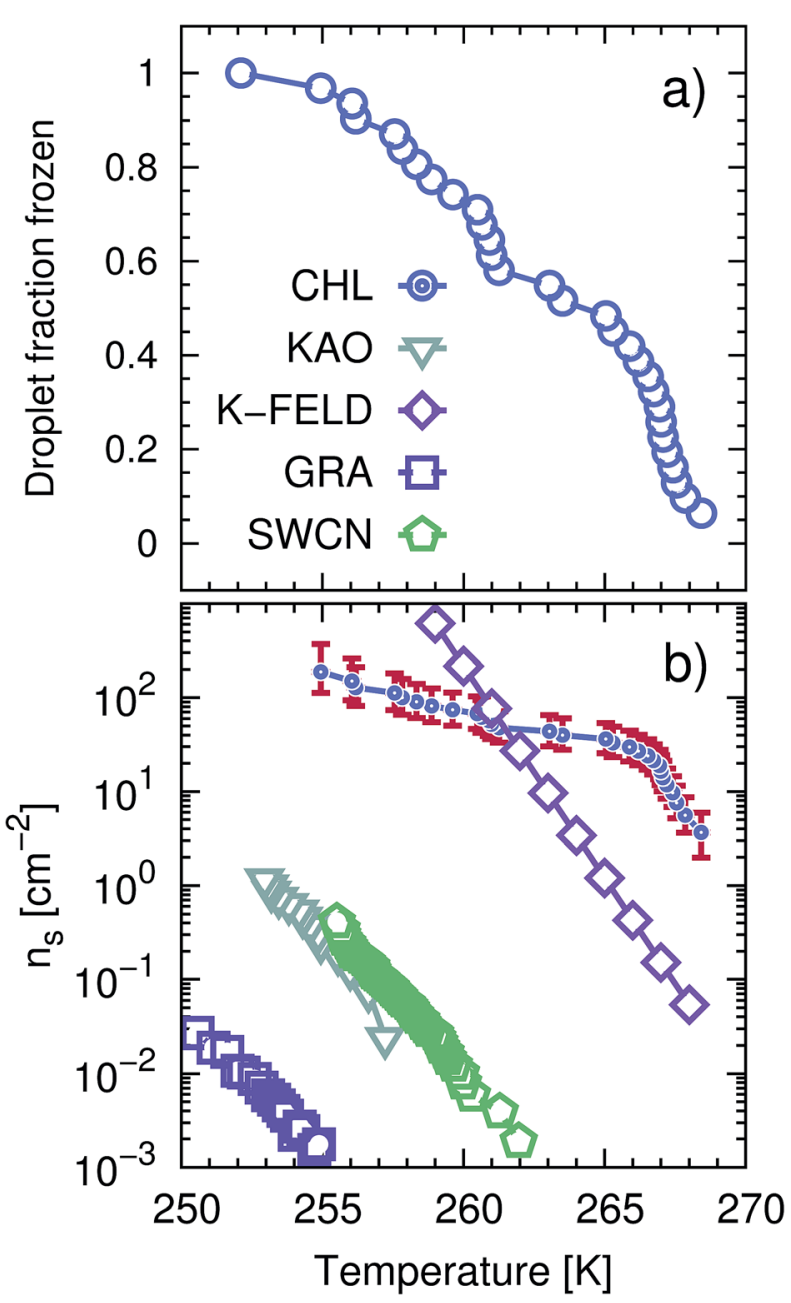

Fig. 1 CHLM crystals promote the formation of ice across a wide range of temperatures. (a) Droplet fraction frozen as a function of temperature for $1 \mu \mathrm{l}$ water droplets placed onto a CHLM substrate. (b) Ice-active surface site density $n_{\mathrm{s}}$ (see text and Methods section) values for the same data reported for CHLM (CHL) in panel (a), together with $n_{\mathrm{s}}$ values for kaolinite (KAO) from Herbert et al., ${ }^{62} \mathrm{BCS} 376$ feldspar (KFELD) from Atkinson et al. ${ }^{11}$ graphene oxide (GRA) from Whale et al. ${ }^{63}$ and single-walled carbon nanotubes (SWCN) also from ref. 63. The uncertainty in terms of temperature associated with the CHLM data is $\pm 0.4 \mathrm{~K}$.

The uncertainties associated with the values of $n_{\mathrm{s}}$ have been calculated using Monte-Carlo simulations of possible active ice nucleation sites distributions, propagated with the uncertainty associated with $A$ - as described in Harrison et al. ${ }^{\mathbf{1 2}}$ These simulations generate a list of possible values for the number of active sites per droplet for a given experiment, given the observed freezing data. By repeating this process a great many times, a distribution of the possible active site distributions that can account for the freezing of each droplet is obtained. The error bars for the CHLM $n_{\mathrm{s}}$ data reported in Fig. 1 are generated by propagating this distribution with the uncertainty in surface area of cholesterol per droplet and taking the 95\% confidence interval of the resulting distribution. At high and low temperature ends of the reported data, where the Poisson uncertainty (i.e. the error originating from the Monte-Carlo simulations) is largest, the contribution of the uncertainty in surface area amounts to approximately $25 \%$ of total uncertainty in $n_{\mathrm{s}}(T)$, with the Poisson uncertainty in the active site distribution accounting for the remainder of the error bars.

Scanning electron microscopy (SEM) was performed on CHLM plates. These were mounted on copper tape, then coated with $2 \mathrm{~nm}$ of iridium. SEM was performed with an FEI Nova NanoSEM 450 in high vacuum mode, using an EverhartThornley Detector (ETD).

\subsection{Molecular dynamics simulations}

The computational setup we have used is depicted in Fig. 3a. A single layer of CHL molecules, cleaved along the (001) plane (perpendicular to the normal to the slab) was prepared by starting from the experimental cell parameters and lattice positions. ${ }^{52}$ Specifically, a CHLM crystal system made of two mirroring slabs (intercalated by water molecules, in a ratio of $1: 1)$ was cleaved along the (001) plane. The triclinic symmetry of the system (space group P1) was preserved, and we have constructed a 3 by 3 supercell with in-plane dimensions of 37.17 and $36.57 \AA$. We positioned 1923 water molecules randomly atop this CHLM slab at the density of the TIP4P/Ice model ${ }^{53}$ at $300 \mathrm{~K}$, and expanded the dimension of the simulation cell along
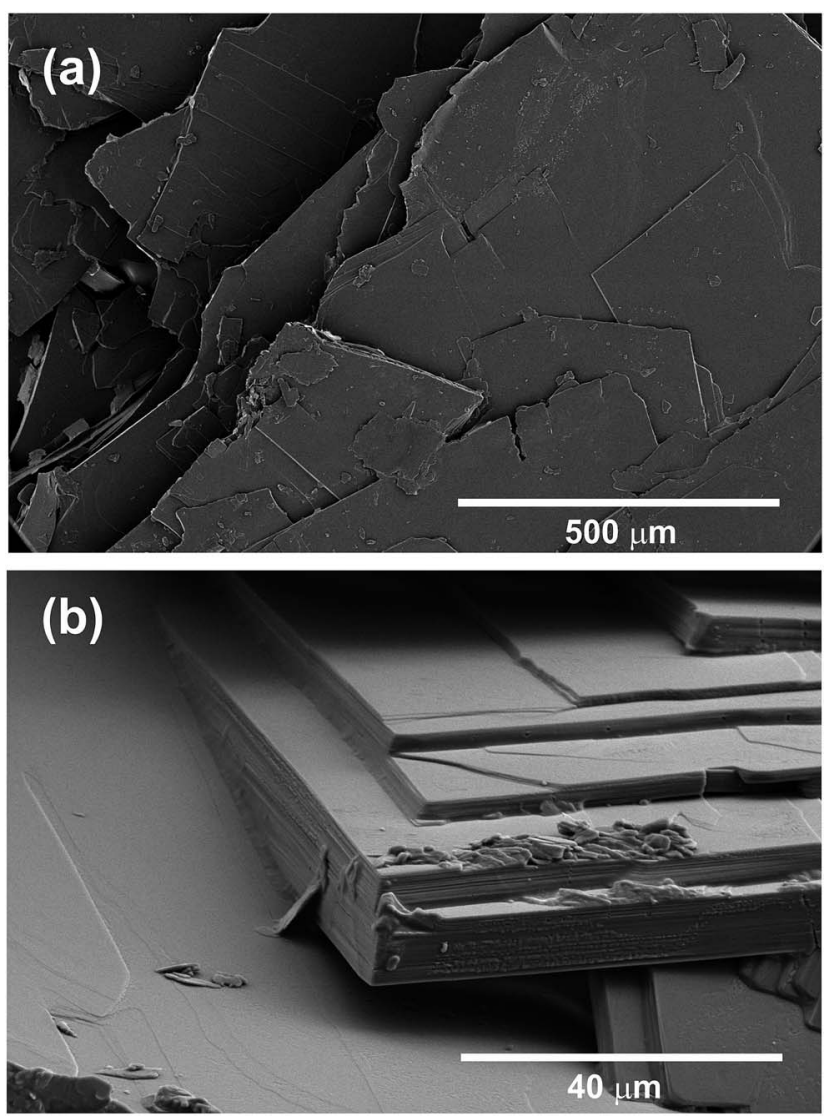

Fig. 2 CHLM crystals display a diverse surface topography. SEM images of CHLM crystals, which predominantly expose $\{001\}$ surfaces - consistent with what has been reported in ref. 54 . It is quite clear that these plates possess numerous topographical features. 

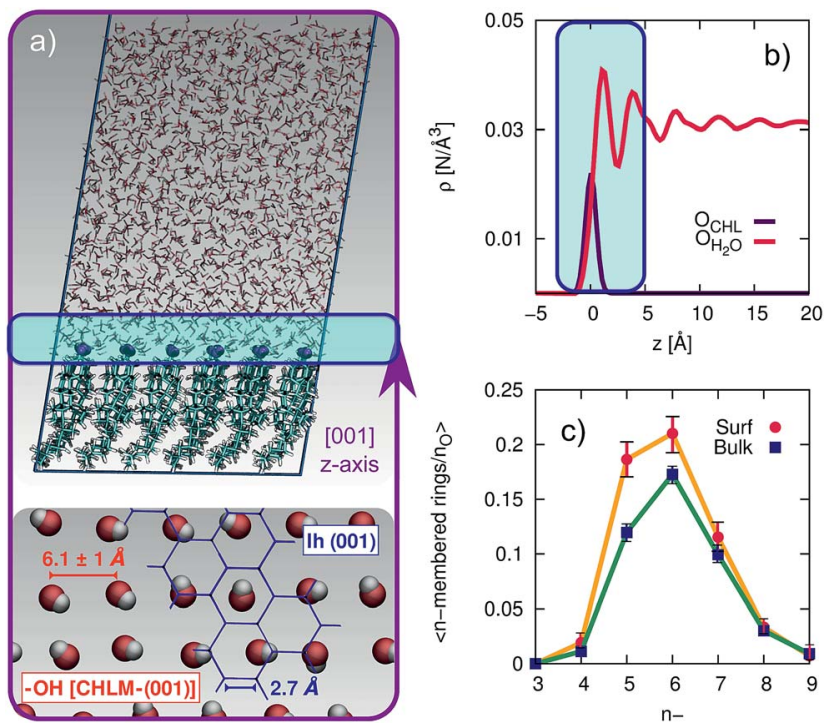

Fig. 3 Structuring of water on the (001) hydroxylated face of CHLM. (a) Representative snapshot of a molecular dynamics simulation of a water slab in contact with the (001) hydroxylated face of CHLM. The inset at the bottom illustrates the arrangement of the hydroxyl group on the CHLM surface; an hypothetical ice $/ \mathrm{h}$ (001) plane (blue) is superimposed on part of the image to highlight the absence of a structural match between -OH groups and ice. (b) Density profile of oxygen atoms belonging to either the $-\mathrm{OH}$ hydroxyl groups of $\mathrm{CHLM}$ molecules $\left(\mathrm{O}_{\mathrm{CHL}}\right)$ or water molecules $\left(\mathrm{OH}_{2} \mathrm{O}\right)$ along the $z$-axis parallel to [001] direction, thus normal to the water-CHLM interface. The zero of the $x$-axis corresponds to the average position of the $\mathrm{O}_{\mathrm{CHL}}$ atoms, while the shaded area in green identifies the water-CHLM interface. Statistics have been accumulated over a $1.5 \mu$ s long simulation at 230 $\mathrm{K}$. (c) Number of $n$-membered rings of hydrogen bonded water molecules at the water-CHLM interface (Surf) or within the bulk of the water slab (Bulk), normalised by the number of oxygen atoms in each region. Note that at the water-CHLM interface oxygen atoms belonging to the $\mathrm{CHLM}-\mathrm{OH}$ hydroxyl groups have also been considered when computing the rings statistics.

the normal to the slab to $100 \AA$. This setup allows for a physically meaningful equilibration of the water at the density of interest at a given temperature, but suffers from two distinct drawbacks: (i) the CHLM slab possesses a net dipole moment which is not compensated throughout the simulation cell and (ii) the presence of the water-vacuum interface can alter the structure and the dynamics of the liquid film. However, we have previously addressed these issues in previous work dealing with the hydroxylated (001) polar surface of the clay mineral kaolinite, ${ }^{44,45}$ concluding that such details do not affect the mechanism nor the kinetics of ice formation. In addition, the water film is thick enough to allow a bulk-like region to exist in terms of both structure and dynamics. The effect of the watervacuum interface is therefore negligible. The slab considered in this work presents the hydrophilic, $-\mathrm{OH}$ terminated heads of the CHL molecules to the water, in agreement with experimental insight. ${ }^{54}$ As we discuss in the main text, the interaction between the hydroxyl groups (which display amphoteric characteristics in terms of the hydrogen bond network) of CHL molecules and water is responsible for the templating effect of CHLM crystals which serves to promote ice nucleation.
The CHARMM36 (ref. 55) force field was used to model the CHL crystals, taking advantage of a recent update of this force field parameters explicitly with respect to CHL. ${ }^{56}$ In order to mimic the experimental conditions, we have constrained the system at the experimental lateral dimensions (detailed together with the computational geometry in the ESI $\dagger$ ), and we have also restrained the positions of the hydrophobic tail of each CHL molecule (specifically, the carbon atoms C25, C26 and C27, see the inset of Fig. S1 in the ESI $\dagger$ ) by means of an harmonic potential characterised by a spring constant of $10000 \mathrm{~kJ} \mathrm{~mol}^{-1}$. All the other atoms within the CHLM slab are unconstrained. We have verified that the thermal expansion of the crystal at $230 \mathrm{~K}(\sim 0.1 \%$ with respect to each lateral dimension) does not alter the structure nor the dynamics of the water-kaolinite interface. This setup is thus as close as we can get to the realistic (001) hydrophilic surface of CHLM within the CHARMM36 model. Implications of the flexibility of the CHLM slab are discussed in the ESI. $\dagger$ The interaction between the water molecules have been modelled using the TIP4P/Ice model, ${ }^{53}$ so that our results are consistent with the homogeneous simulations of ref. 57. The interaction parameters between the clay and the water were obtained using the standard Lorentz-Berthelot mixing rules. ${ }^{58}$

Extreme care must be taken in order to correctly reproduce the structure and the dynamics of the water-CHLM interface. The Forward Flux Sampling (FFS) simulations reported in this work rely on a massive collection of unbiased Molecular Dynamics (MD) runs, all of which have been performed using the GROMACS package, version 4.6.7. The code was compiled in singleprecision, in order to alleviate the huge computational workload needed to converge the FFS algorithm and because we have taken advantage of GPU acceleration, which is not available in the double-precision version. The equations of motions were integrated using a leap-frog integrator with a time step of $2 \mathrm{fs}$. The van der Waals (non bonded) interactions were considered up to $10 \AA$, where a switching function was used to bring them to zero at 12 $\AA$. Electrostatic interactions have been dealt with by means of an Ewald summation up to $12 \AA$. The NVT ensemble was sampled at $230 \mathrm{~K}$ using a stochastic velocity rescaling thermostat ${ }^{59}$ with a very weak coupling constant of 4 ps in order to avoid temperature gradients throughout the system. The geometry of the water molecules (TIP4P/Ice being a rigid model) was constrained using the SETTLE algorithm ${ }^{60}$ while the P_LINCS algorithm ${ }^{61}$ was used to constrain the $\mathrm{O}-\mathrm{H}$ bonds within the clay. We have verified that these settings reproduce the dynamical properties of water reported in ref. 57 . The system was equilibrated at $300 \mathrm{~K}$ for $10 \mathrm{~ns}$, before being quenched to $230 \mathrm{~K}$ over $50 \mathrm{~ns}$. This is the starting point for the calculation of the initial flux rate for the FFS algorithm, which lasted about $1.5 \mu \mathrm{s}$ and thus allowed us to investigate the water-CHLM interface as well (see e.g. Fig. 3).

\section{Results}

\subsection{Cholesterol promotes the formation of ice across a wide range of temperatures}

We start by experimentally investigating the ice nucleating ability of cholesterol crystals - as a function of supercooling. 
CHL can crystallise into two different polymorphs, namely anhydrous $^{64}$ (CHLA) and CHLM. ${ }^{52}$ The latter is the most relevant to ice formation, as it spontaneously forms in aqueous environments. ${ }^{52,54,65-68}$ Conveniently, CHLM crystallises from a mixture of $95 \%$ ethanol and $5 \%$ water as plates with the (001) surface forming the flat surface of the plates. ${ }^{66}$ The platy crystal habit of CHLM is characteristic of CHLM as opposed to CHLA, which tends to crystallise as needles. CHLM crystals display a layered structure: bilayers of CHL molecules are stacked along the [001] direction, and facile cleavage along the (001) planes leads to surfaces exposing either a $-\mathrm{CH}_{3}$ terminated, hydrophobic surface or a $-\mathrm{OH}$ terminated, hydrophilic surface. Atomic and chemical force microscopy measurements indicate that in aqueous and organic solution conditions, the hydrophilic (001) surface is most abundantly found, in the form of largely homogeneous crystalline faces. ${ }^{54}$ Early experimental evidence suggested substantial ice nucleation activity of CHLM at very mild supercooling $\left(\Delta T_{\mathrm{S}}=5 \mathrm{~K}\right) .^{21,23,69}$ The ice nucleation efficiency of CHLM was evaluated using an adapted version of the $\mu \mathrm{l}$-Nucleation by Immersed Particles Instrument ( $\mu \mathrm{l}$-NIPI) experiments $\mu \mathrm{l}$-NIPI described in detail in ref. 50. Experiments were performed by placing droplets directly onto a surface of crystalline CHLM. We used an electronic pipette to place $1 \mu \mathrm{l}$ droplets of water onto the (001) plane of plates of CHLM. The water droplets were then cooled down at a rate of 1 $\mathrm{K} \min ^{-1}$ and freezing monitored using a camera. In this way the fraction of frozen droplets can be determined as a function of temperature. Note that as the crystalline surface is submerged in liquid water these experiments are conducted at $100 \%$ relative humidity - i.e. in "immersion mode". ${ }^{70}$

In Fig. 1a we report the fraction of frozen droplets as a function of temperature for CHLM. It can be seen that CHLM can induce ice nucleation at temperatures as warm as $269 \mathrm{~K}$. This agrees with previous studies which have reported high nucleation temperatures for CHL in the immersion mode., ${ }^{2,2}$ In here, we investigate the ice nucleating ability of CHLM as a function of supercooling. As shown in Fig. 1a, the spread of nucleation temperatures for the CHLM sheets is very broad, with some of them freezing at temperatures as low as $252 \mathrm{~K}$. To allow for a comparison of the efficiency of ice nucleation by CHLM with other known nucleating species we have calculated the ice-active surface site density $\left(n_{\mathrm{s}}\right)$ for CHLM on the basis of the size of the contact patch of the water droplets with the CHL plates. As explained in greater detail in the Methods section, $n_{\mathrm{s}}$ is a site specific measure of ice nucleation efficiency which does not take into account the time dependence of ice nucleation, on the basis that the impact of time dependence on heterogeneous ice nucleation is generally minimal. ${ }^{62,70,71} \mathrm{We}$ have compared the ice nucleating efficiency of CHLM with that of e.g. kaolinite powder ${ }^{62}$ which has commonly been regarded as an efficient ice nucleating agent in the past ${ }^{72}$ and of BCS 376 feldspar powder, which is known to nucleate ice highly efficiently ${ }^{11}$ and was likely responsible for earlier observations of efficient ice nucleation in kaolinite samples. It can be seen that CHLM nucleates ice far more efficiently than kaolinite and more efficiently even than the feldspar at warm temperatures. Thus, CHLM has the potential to be a highly efficient ice nucleating agent in immersion mode across a wide range of temperatures - which is the scenario typically encountered when dealing with cryobiological applications.

\subsection{The role of surface topography}

As noted above the spread of freezing temperatures we report for CHLM is very broad. This behaviour suggests that the nucleation behaviour of CHLM is spatially heterogeneous, i.e. different parts of the surface nucleate ice with differing efficiency. This is commonly known as site specific nucleation behaviour, ${ }^{62,70,71,73}$ and it can be appreciated to a lesser extent for the other ice nucleating agents considered in Fig. 1b. However, it is interesting to note that CHLM crystals seem to lead to two different ice nucleation regimes, as can be inferred from Fig. $1 \mathrm{~b}$ (note the two different slopes characterising $n_{\mathrm{s}}$ as a function of temperature). As it is becoming increasingly clear that the topography of the ice nucleating agents must play an important role in the heterogeneous nucleation of ice from liquid water, ${ }^{40,74,75}$ we suspect that structural differences between the crystalline areas covered by the water droplets are responsible for the wide spread in nucleation temperatures observed. This hypothesis is supported by the scanning electron microscope (SEM) images of the (001) face of CHLM reported in Fig. 2. While the crystalline plates appear as mostly flat and smooth within the resolution of $\sim 100 \mu \mathrm{m}$, it is clear that there exist numerous defects, which can potentially present opportunities for complicated surface geometries to occur. How exactly the nanometric structure of crystalline ice nucleating agents affects the kinetics of ice formation is still an open question (see e.g. ref. 40 and 32). In fact, it would be expected that an atomically smooth and homogeneous CHL surface would nucleate ice with a single nucleation rate and hence within a far narrower range of temperatures than that reported in Fig. 1. The role of specific defects and broadly speaking of the surface morphology to ice formation on CHL - and the vast majority of biological nucleating agents - it thus remains yet to be fully understood. For instance, it is not immediately clear why CHLM crystals are much more effective than feldspar in promoting the formation of ice. In the next section, we will address this issue by showing that in addition to the topography of the surface, the formation of a peculiar hydrogen bond network at the water-CHLM interface is key in determining the ice nucleating ability of this compound.

\subsection{The cholesterol-water interface}

In order to investigate the molecular-level details of the CHLMwater interface, we have performed unbiased molecular dynamics simulations at strong supercooling $\left(\Delta T_{\mathrm{S}}=42 \mathrm{~K}\right)$ employing the CHARMM36 (ref. 55 and 56) and the TIP4P/Ice ${ }^{53}$ force fields for $\mathrm{CHL}$ and water molecules respectively. Computational details and results concerning the validation of our computational setup are reported in the Methods section and in the ESI $\dagger$ respectively, while the computational geometry is depicted in Fig. 3a.

A water slab ( $\sim 40 \AA$ thick) is in contact with the hydroxylated (001) surface of CHLM ( $\left.\mathrm{CHLM}_{001}^{-\mathrm{OH}}\right)$, modelled as a single layer of CHL molecules. This surface is hydrophilic, due to the presence 
of amphoteric hydroxyl groups. As CHL molecules are relatively bulky and the crystal is held together by weak electrostatic interactions only, the arrangement of these $-\mathrm{OH}$ groups on the CHLM surface is characterised by a broad distribution of large $\mathrm{OH}-\mathrm{OH}$ distances, ranging from 5.1 to $7.1 \AA$ - as illustrated in Fig. 3a. Such a pattern of hydroxyl groups does not straightforwardly match any particular low-index Miller surface of either hexagonal or cubic ice. This is relevant, as a good structural match between a substrate and ice ${ }^{38}$ has traditionally been considered as a "requirement" of an effective ice nucleating agent. $^{76}$

Interestingly, despite the presence of the hydroxyl groups, the density profile of the oxygen atoms of the water molecules on CHLM reported in Fig. 3b resembles that for water at hydrophobic walls. ${ }^{77}$ The enhancement $(\sim 30 \%$ in Fig. $3 \mathrm{~b})$ of the density, within the first peak of the profile, compared to its value in the bulk of the water slab, is much smaller than that (typically a factor four or six) observed for e.g. water in contact with hydrophilic walls - or indeed water on kaolinite. This is because, the outer layer of the CHLM crystals is much more mobile/flexible than that of kaolinite: this is not surprising, as we are comparing a molecular organic crystal (held together by van der Waals interactions) with a (covalently bonded) clay mineral. Importantly, it is reasonable to assume that a similar degree of flexibility characterises the majority of organic crystals containing long molecules such as steroids. This is relevant to ice formation because, as discussed in e.g. ref. 43, the structural fluctuations of organic/biological ice nucleating particles can strongly affect the kinetics of ice nucleation. In fact, we have shown in ref. 45 that the same argument holds in the case of kaolinite as well: for instance, a "frozen" kaolinite surface (atoms are kept fixed during MD simulations) leads to nonphysically fast ice nucleation rates.

Because of this flexibility of the CHL molecules and the lowdensity of hydroxyl groups at the water-CHLM interface, we did not observe (within a $200 \mathrm{~ns}$ time scale) the formation of an ordered, ice-like over-layer of water molecules, in contrast with what is generally found in the case of idealised crystalline surfaces ${ }^{78}$ carbonaceous particles ${ }^{42}$ or kaolinite crystals. ${ }^{44,45}$ In fact, most inorganic substrates are characterised by surfaces where atomic/molecular species are tightly packed, and can thus potentially provide a high density of functional groups for supercooled water to interact with, typically by forming a more or less ordered overlayer sitting on top of the crystalline surface. In the case of the water-CHLM interface, however, water molecules can partially infiltrate the outer layer of the CHLM surface (see Fig. 3a and b) due to the relatively large spacing between the CHL molecules and the flexibility of the surface itself. As a net result, despite the absence of a flat overlayer of ice-like water molecules, the amphoteric character of the hydroxyl groups does facilitate the formation of a network of hydrogen bonded rings of water molecules as well as hydroxyl groups, as illustrated in Fig. 3c. In particular, we observe the emergence of 6-membered rings of hydrogen bonded water molecules and hydroxyl groups. These rings are the building blocks of both hexagonal (ice $I_{\mathrm{h}}$ ) and cubic (ice $I_{\mathrm{c}}$ ) ice, and are the most abundant species in bulk water. Note that the occurrence of these rings is actually even more pronounced in the proximity of the CHLM-water interface (red/orange points/ curve in Fig. 3c). Surprisingly, there is also an increase in the number of 5-membered water/hydroxyl rings at the crystalliquid interface. Pentagonal rings are thought to frustrate the homogeneous formation of ice $;^{79}$ however, in this case both 6and 5-membered rings alike contribute to the formation of icelike fluctuations such as the "cage" shown in Fig. 4a. These cages are indeed the building blocks of ice $I_{\mathrm{c}}$ (see Fig. 4b and c), and involve hydrogen bonds between water molecules and hydroxyl groups, as depicted in Fig. 4d and e. Thus, in this heterogeneous nucleation scenario, the presence of 5membered rings in not detrimental; on the contrary, they lead to the formation of ice-like fluctuations of the water network at the water-CHLM $\mathrm{COH}_{001}^{-\mathrm{OH}}$ interface.

We note that the emergence of these cages is the reason why we have chosen to consider as the "interfacial layer" those water
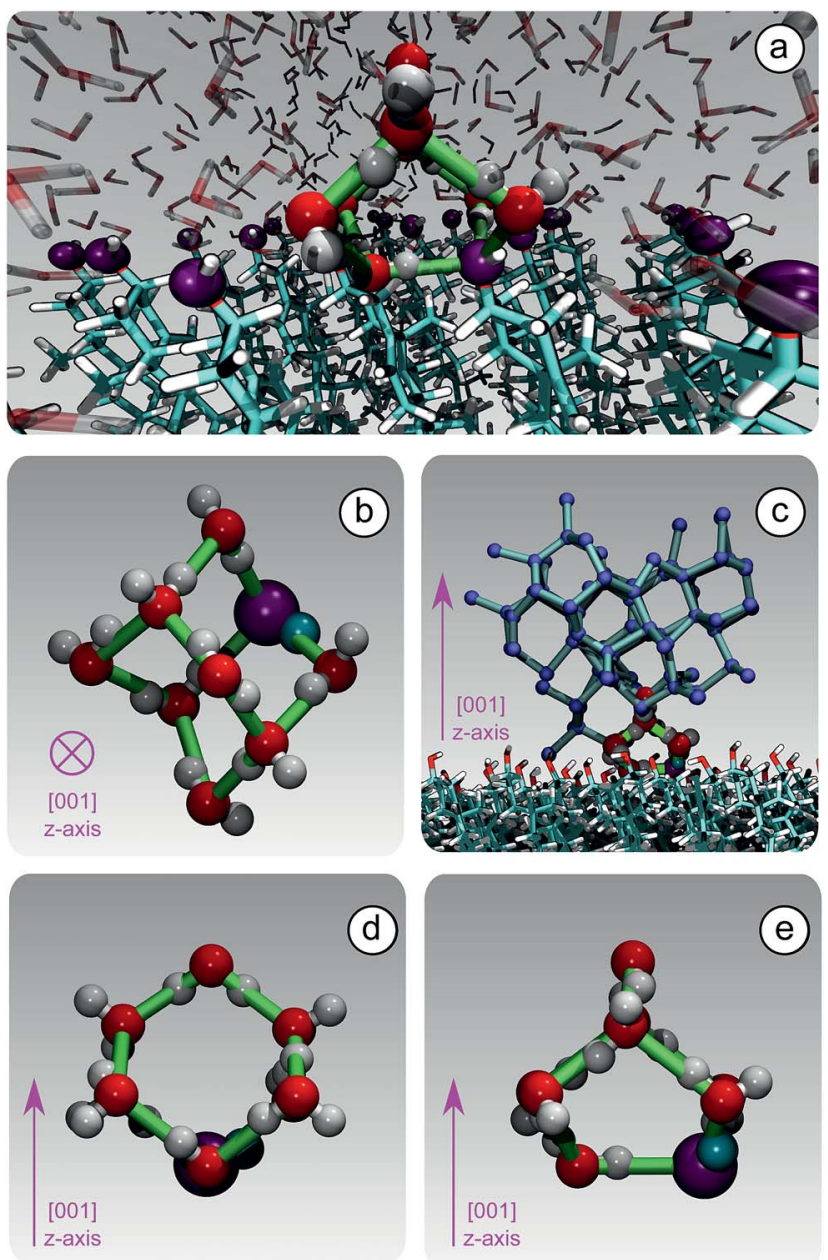

Fig. 4 The formation of unconventional ice-templating molecular structures: hydrogen bonded cages. (a) A hydroxyl group (purple) of CHLM participates into a hydrogen bonded cage of water molecules. (b) A single hydrogen bonded cage (top view), which constitutes the building block of cubic ice (panel (c), side view). Note that these cages are made of both 6-membered (panel (d), side view) and 5-membered (panel (e), side view) hydrogen bonded rings, involving water molecules as well as a hydroxyl group provided by the CHLM surface. 
molecules within $5.0 \AA$ from the average position of the oxygens of the CHL hydroxyl groups - as illustrated by the shaded region in Fig. 3b. As shown in e.g. ref. 80, the definition of this water layer can have an impact on the analysis of the structure of - in this case - the water-CHLM interface. While the interfacial layer can be chosen on the basis on indicators such as the first or second minimum of the density profile (see Fig. $3 \mathrm{~b}$ ), we have found that the rather generous cutoff of $5.0 \AA$ is sufficient to accommodate the substantial extent of the hydrogen bonded cages depicted in Fig. $4 \mathrm{~d}$ and $3 \mathrm{e}$. We have also verified that by choosing the second minimum of the density profile $(\sim 7 \AA)$ our results, including the trends within the rings statistics reported in Fig. 3c, are basically unchanged.

Our findings thus contribute the growing body of evidence $^{40,43,78}$ that the structural mismatch argument alone cannot be deemed as neither a sufficient nor a necessary criteria to assess, let alone to predict, the ice nucleating ability of a given substrate. ${ }^{38}$ This is bound to be especially true in the case of biological ice nucleating agents such as macromolecules, ${ }^{17}$ where the notion itself of a lattice mismatch is ill defined. In fact, we argue that organic crystals such as cholesterol lie halfway in between inorganic (e.g. mineral crystals) and biological (e.g. bacterial fragments) ice nucleating agents, as they are characterised by the relatively flat and (in this case) -OH regularly patterned surfaces of the former while showing the flexibility of the latter. This is particularly relevant for CHL, which is a substantial component of animal cellular membranes ${ }^{25}$ and could thus contribute to promote the heterogeneous formation of ice in biological matter - a possibility we will investigate in future work. In this respect, it is interesting to note that very recent simulations ${ }^{81}$ suggest that ice can bind to antifreeze proteins via "anchored clathrate" motifs not dissimilar to the molecular cages discussed above.

\subsection{Ice nucleation mechanism and kinetics}

In order to characterise the mechanism as well as the kinetics of ice nucleation on $\mathrm{CHLM}_{001}^{-\mathrm{OH}}$ we have performed forward flux sampling (FFS) simulations. ${ }^{82-89}$ While other enhanced sampling techniques are in principle available, such as metadynamics ${ }^{90}$ transition path sampling, ${ }^{91}$ and seeded molecular dynamics ${ }^{92}$ FFS represents a "gold standard" approach when dealing with ice nucleation (see e.g. ref. 35, 44, 57, 89 and 93). This method involves partitioning the path from (in this case) liquid water to ice, described by an order parameter $\lambda$, into a collection of interfaces $\lambda_{\mathrm{i}}$. Here, $\lambda$ corresponds to the number of water molecules within the largest ice nucleus, which can be located either in the bulk of the water slab or at the water$\mathrm{CHLM}_{001}^{-\mathrm{OH}}$ interface. A diffuse crystal-liquid interface has been taken into account into the definition of $\lambda$, which relies on local bond order parameters (see ESI $\dagger$ and ref. 94), consistent with ref. 44. Starting from the natural fluctuations of liquid water toward the ice phase, i.e. pre-critical ice nuclei as sampled within $\mu$ s long unbiased MD simulations, the nucleation rate $J$ can be obtained as the product of the flux $\Phi_{\lambda_{0}}$ by which the system reached the first interface $\lambda_{0}$, times the product of the sequence of the individual crossing probabilities $P\left(\lambda_{\mathrm{i}} \mid \lambda_{\mathrm{i}-1}\right)$ :

$$
J=\Phi_{\lambda_{0}} \prod_{i=1}^{N_{\lambda}} P\left(\lambda_{\mathrm{i}} \mid \lambda_{\mathrm{i}-1}\right)
$$

In this way, the (exceedingly small) total probability $P\left(\lambda_{\text {ice }} \mid \lambda_{0}\right)$ for a certain MD trajectory to reach the ice basin is decomposed in a collection of (manageable) crossing probabilities which we compute by a large number $\left(10^{3}\right.$ to $\left.10^{5}\right)$ of unbiased MD trial runs from $\lambda_{\mathrm{i}-1}$ to $\lambda_{\mathrm{i}}$. Further details about the FFS algorithm can be found in the ESI. $\dagger$ We note that we have used the same water model (TIP4P/Ice) at the same strong supercooling $\left(\Delta T_{\mathrm{S}}=42 \mathrm{~K}\right)$ as employed previously to compute the homogeneous ice nucleation rate and the heterogeneous ice nucleation rate on kaolinite, a clay mineral of relevance to atmospheric science. As such, we can compare directly our results with those of ref. 44 and 57.

From the very early stages of the nucleation process, we observe a strong preference for ice to form at the water$\mathrm{CHLM}_{001}^{-\mathrm{OH}}$ interface - as opposed to within the bulk of the water slab. In fact, $\sim 75 \%$ of the pre-critical ice nuclei we observe as natural fluctuations of the supercooled water network $(\lambda=0)$ sit on top of the $\mathrm{CHLM}_{001}^{-\mathrm{OH}}$ surface. The calculated growth probability $P\left(\lambda \mid \lambda_{0}\right)$ as a function of lambda, together with the fraction of ice nuclei that can be found at the water-CHLM ${ }_{001}^{-\mathrm{OH}}$ interface are reported in the ESI (Fig. S2b $†$ ). By the time the FFS algorithm has reached $\lambda=125$, no nuclei within the bulk of the water slab survive. We have observed a similar trend in the case of ice nucleation on the hydroxylated (001) basal face of kaolinite, ${ }^{44}$ but the fraction of ice nuclei at the water-kaolinite interface at the initial stages of the FFS algorithm was much smaller $(\sim 25 \%)$. This suggests that pre-critical ice-like fluctuations, which we have recently investigated in the broader context of heterogeneous crystal nucleation, ${ }^{95}$ are much more likely to occur at the surface of CHLM compared to kaolinite.

The mechanism of ice nucleation at the water$\mathrm{CHLM}_{001}^{-\mathrm{OH}}$ interface is illustrated in Fig. 5: the early stages involve the formation of elongated, almost one dimensional, linear, chain-like ice nuclei preferentially along specific directions (see $\mathrm{ESI} \dagger$ ), due to the particular arrangement of the $-\mathrm{OH}$ hydroxyl groups on the $\mathrm{CHLM}_{001}^{-\mathrm{OH}}$ surface. However, larger nuclei (corresponding to increasing values of $\lambda$ ) progressively assume a more isotropic shape, as indicated by the evolution of the asphericity parameter $\alpha$ (equal to 1 and 0 for a infinitely elongated rod and a perfect sphere respectively) as a function of $\lambda$. At the same time, the $1 \mathrm{D}$ character of the nuclei evolves toward a more compact geometry, with a significant growth along the [001] direction (z-axis) normal to the water$\mathrm{CHLM}_{001}^{-\mathrm{OH}}$ interface, as demonstrated by the trend of the dimension $\Delta Z$ of the ice nuclei along that axis, also reported in Fig. 5. The resulting morphology of the ice crystals, though, remains to be investigated because of the emergence of finite size effects. Overall, the evolution of the ice nuclei within the early stage of ice nucleation at the water- $\mathrm{CHLM}_{001}^{-\mathrm{OH}}$ interface possesses some similarities with the case of ice formation on kaolinite ${ }^{44}$ where ice nuclei spread into a 2D, planar geometry before stacking additional ice layers along the normal to the 


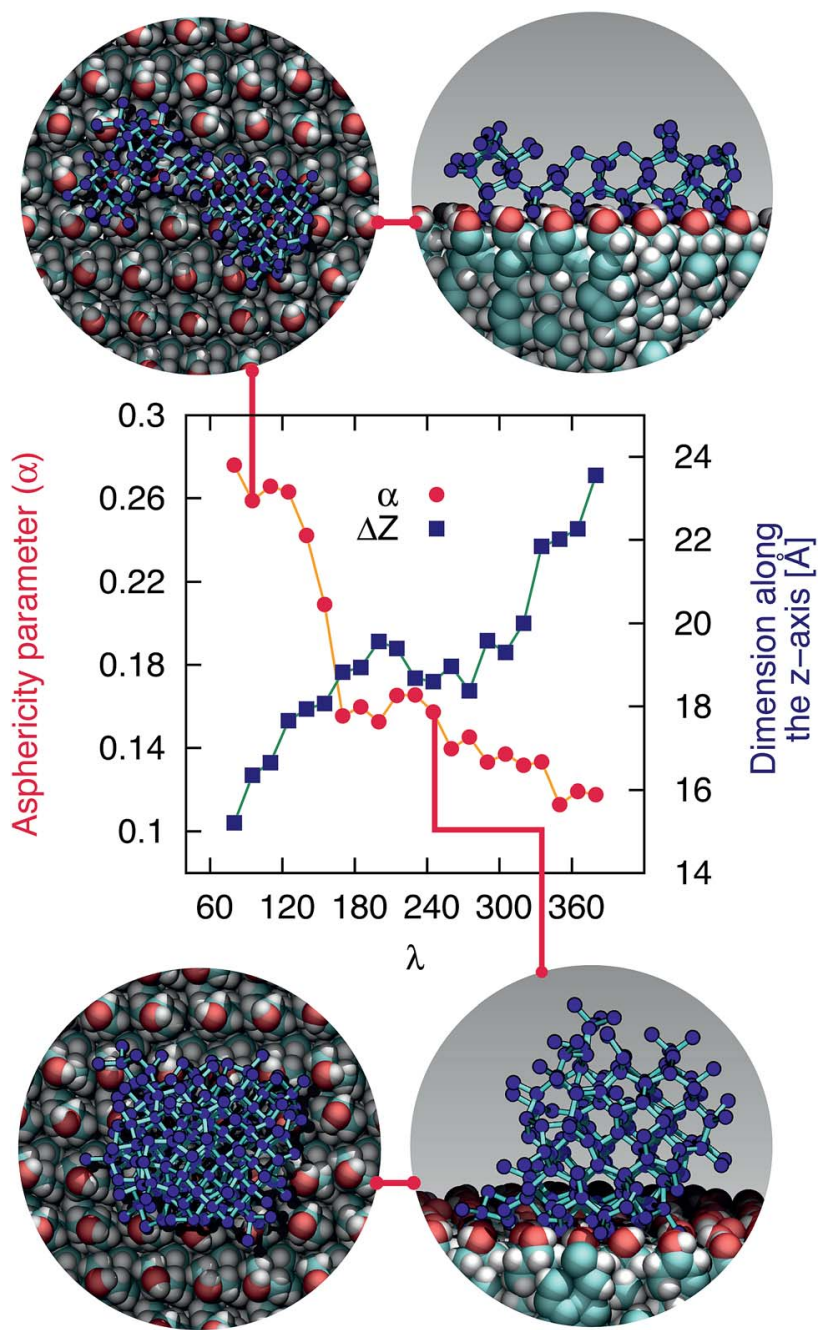

Fig. 5 The early stages of ice nucleation at the water$\mathrm{CHLM}_{001}^{-\mathrm{OH}}$ interface involve non-spherical ice crystallites. Asphericity parameter $\alpha$ and spatial extent of the ice nuclei along the direction normal to the CHLM slab $\Delta Z$ as a function of $\lambda$ for ice nuclei at the water-CHLM $\mathrm{OOH}^{-\mathrm{OH}}$ interface. The insets correspond to top and side views of typical ice nuclei forming at the water-CHLM $\mathrm{COO}_{01}^{-\mathrm{O}}$ interface, containing about 100 (top) and 245 (bottom) water molecules.

water-kaolinite interface, once the critical nucleus has been reached. Thus, these findings suggest that the nature of the early stages of heterogeneous ice nucleation at strong supercooling $\left(\Delta T_{\mathrm{S}}=42 \mathrm{~K}\right)$ has a strong anisotropic character, in stark contrast with the assumptions prescribed by classical nucleation theory (CNT). ${ }^{96}$

In fact, CNT does not take into account the molecular structure nor the "chemistry" of the substrate: these aspects are only implicitly included into the value of the contact angle of the ice nuclei with respect to the substrate. However, microscopic features such as the particular arrangement of the hydroxyl groups on the CHLM surface can influence the shape and the energetics of the ice nuclei. In the case of ice on CHLM, water molecules at the water-cholesterol interface find convenient to harness the directionality of the $-\mathrm{OH}$ pattern (see Fig. $3 \mathrm{a}$ and 3 in the ESI $\dagger$ ) to form anisotropic ice nuclei (see
Fig. 5 and 3 in the ESI $\dagger$ ), which are likely to be characterised by a much smaller interfacial energy if compared to the hemispherical shape predicated by CNT in the case of perfectly flat, featureless substrates. We note however that in order to probe this aspect of CNT quantitatively, it would be desirable to improve the current enhanced sampling techniques to take into account milder supercooling - and thus larger critical ice nuclei.

The ice nucleation rate on the $\mathrm{CHLM}_{001}^{-\mathrm{OH}}$ surface obtained from our FFS simulations is $10^{27 \pm 3} \mathrm{~s}^{-1} \mathrm{~m}^{-3}$, about 20 orders of magnitude larger than the homogeneous ice nucleation rate at the same supercooling - calculated via FFS simulations using the same water model. ${ }^{57}$ This spectacular enhancement of the kinetics of ice formation is due to the small heterogeneous critical nucleus size $\mathrm{N}_{\mathrm{H}}^{*}$, which we estimate (as discussed in detail in the ESI $\dagger$ ) to contain $250 \pm 50$ water molecules a number consistent with the predictions of CNT (see ESI $\dagger$ and ref. 44). Interestingly, these results are very similar to what we have previously obtained in the case of ice formation on kaolinite, ${ }^{44}$ where we calculated $J=10^{26 \pm 2} \mathrm{~s}^{-1} \mathrm{~m}^{-3}$ and $\mathrm{N}_{\mathrm{H}}^{*}=$ $225 \pm 25$. However, it has to be said that the FFS simulations performed in this work (as opposed to the case of kaolinite ${ }^{\mathbf{4 4}}$ ) may be suffering from finite size effects (discussed in the $\mathrm{ESI}_{\dagger} \dagger$ ), which could both enhance the kinetics of ice nucleation (as the ice nuclei feel the influence of their periodic images) and/or hamper the growth of ice crystals (as the simulation box most likely does not match the periodicity of the growing ice crystal). Our estimates of $J$ and $\mathrm{N}_{\mathrm{H}}^{*}$ have therefore to be taken with care.

The fact that the kinetics of ice formation on the $\mathrm{CHLM}_{001}^{-\mathrm{OH}}$ surface seems to be comparable with that of an inorganic crystal such as kaolinite is not entirely unexpected, as the (001) hydroxylated surface of kaolinite also presents $-\mathrm{OH}$ groups at the water-crystal interface which are capable of templating the formation of ice-like structures. However, supercooled water on kaolinite forms a dense, hexagonal ordered overlayer of ice-like molecules sitting on top of the hydroxyl groups, ${ }^{45}$ while, as we have discussed in the previous section, water molecules can partially infiltrate the $\mathrm{CHLM}_{001}^{-\mathrm{OH}}$ surface to form 5- and 6-membered hydrogen bonded rings, resulting in a much less ordered and way less dense overlayer. As both substrates (kaolinite and CHLM) are characterised by the presence of hydroxyl groups which facilitate the formation of ice, the much faster kinetics of ice nucleation we have observed experimentally for CHLM compared to kaolinite (especially at mild supercooling, Fig. 1b) is likely to be due to the different surface topography of the two compounds.

\subsection{Competition between cubic and hexagonal ice}

At the strong supercooling considered here $\left(\Delta T_{\mathrm{S}}=42 \mathrm{~K}\right)$, homogeneous ice nucleation results in a mixture of ice $I_{\mathrm{c}}$ and ice $I_{\mathrm{h}}$ known as stacking disordered ice $I_{\mathrm{sd}} \cdot{ }^{97-99}$ However, things can be quite different in the heterogeneous case. For instance, the hydroxylated (001) basal face of kaolinite promotes exclusively the formation of the primary prism face of ice $I_{\mathrm{h}} \cdot{ }^{\mathbf{4 4 , 4 5}}$ In the case of the $\mathrm{CHLM}_{001}^{-\mathrm{OH}}$ surface, we observe both ice $I_{\mathrm{c}}$ and ice $I_{\mathrm{h}}$ 

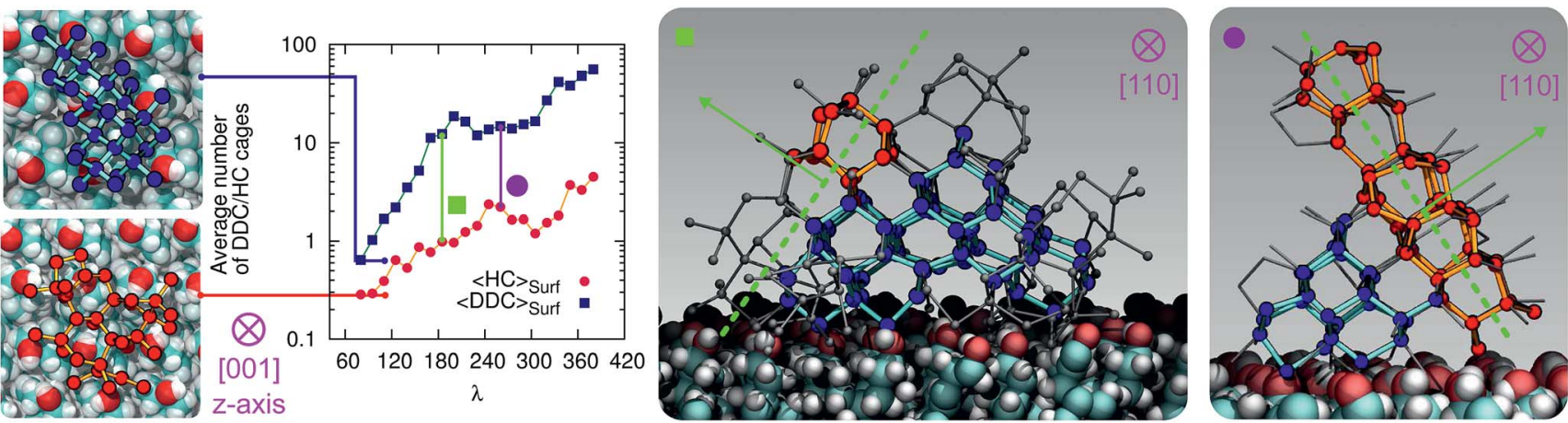

Fig. 6 Competition between cubic (blue/cyan spheres/sticks) and hexagonal (red/orange spheres/sticks) ice within the early stage of ice nucleation at the water-CHLM $\mathrm{OO1}_{01}^{-\mathrm{H}}$ interface. The average number of double diamond and hexagonal cages (DDC and $\mathrm{HC}$, the building block of ice $I_{\mathrm{c}}$ and ice $I_{\mathrm{h}}$ respectively) is reported as a function of the order parameter $\lambda$. Insets on the left show representative ice $I_{\mathrm{C}}$ and ice $I_{\mathrm{h}}$ fluctuations (top view) at the first FFS interface $(\lambda=80)$. Insets on the right show representative ice nuclei at $\lambda=165$ and 260 , where the competition between the two polymorphs becomes more evident. The dashed (green) lines/arrows indicate the crystallographic plane/direction along with ice $I_{\mathrm{h}}$ has the possibility to grow on top of ice $I_{\mathrm{c}}$.

nuclei at the very early stages of the nucleation process, as depicted in the inset (left side) of Fig. 6. These ice-like fluctuations originate from the templating effect of the hydroxyl groups on the CHLM surface, as illustrated in Fig. 4a-e (and Fig. S3 in the ESI $\dagger$ ). In principle, even if ice $I_{\mathrm{c}}$ nuclei are three times more abundant than ice $I_{\mathrm{h}}$ nuclei at the first FFS interface $(\lambda=80)$, we would expect the formation of ice on the $\mathrm{CHLM}_{001}^{-\mathrm{OH}}$ surface to proceed via the growth of ice $I_{\mathrm{sd}}$. In fact, as shown in Fig. 6, the competition between the growth of two ice polytypes at $230 \mathrm{~K}$ (i.e. $\Delta T=42 \mathrm{~K}$ ) is dominated by ice $I_{\mathrm{c}}$ : by the time the ice nuclei have reached a post-critical size (e.g. $\lambda=360)$, the average number of Hexagonal Cages (HC, ${ }^{57}$ the building blocks of ice $I_{\mathrm{h}}$ ) is still about three times larger than that of DDC (Double Diamond Cages,${ }^{57}$ the building blocks of ice $I_{\mathrm{c}}$ ).

Interestingly, despite the predominance of ice $I_{\mathrm{c}}$ within the growing ice nuclei, ice $I_{\mathrm{h}}$ can still form and grow along a specific direction (the [111] of the cubic phase) on top of ice $I_{\mathrm{c}}$ crystals (which in turn grow along the [100] direction, normal to the plane of the water- $\mathrm{CHLM}_{001}^{-\mathrm{OH}}$ interface), as illustrated in the insets (right side) of Fig. 6. The coexistence of ice $I_{\mathrm{c}}$ and ice $I_{\mathrm{h}}$ is thus likely to result in ice $I_{\mathrm{sd}}$ crystals at strong supercooling. However, at milder supercooling ice $I_{\mathrm{h}}$ fluctuations are expected to become more relevant, and in fact experimental evidence suggests that the macroscopic crystalline habit of ice crystals grown on CHLM at $\Delta T=2 \mathrm{~K}$ is indeed that of ice $I_{\mathrm{h}}{ }^{22}$ Importantly, we did not observe such a competition between ice $I_{\mathrm{c}}$ and ice $I_{\mathrm{h}}$ in the case of kaolinite, where the cubic polytype is basically absent throughout the whole nucleation process. ${ }^{44,45}$ In fact, we argue that, in the case of CHLM crystals, different nucleation sites (whose exact nature remains to be determined) could promote chiefly ice $I_{\mathrm{c}}$ or ice $I_{\mathrm{h}}$ according to the different degree of supercooling, thus contributing to unravel the strong ice nucleating ability of CHLM crystals along such a wide range of temperatures. This argument would suggest that the multi-component nature of ice nucleation on biological matter could be at least partially attributed to a greater variety of nucleation sites - as well as the specific templating effect of functional groups acting as hydrogen bond donor and/or acceptors with respect to supercooled liquid water. Moreover, we have shown in this work that some of these functional groups - such as the hydroxyl groups characterising the water- $\mathrm{CHLM}_{001}^{-\mathrm{OH}}$ interface - can even promote a different ice polytype at the same time, possibly according to different supercooling.

Finally, we note that, in agreement with previous simulations of ice nucleation, ${ }^{43,45}$ the flexibility of the $\mathrm{CHLM}_{001}^{-\mathrm{OH}}$ has an impact on the extent and the structure of the ice-like fluctuations at the $\mathrm{CHLM}_{001}^{-\mathrm{OH}}$-water interface, and that the anhydrous crystalline phase of CHL also displays substantial ice nucleating potential. These two aspects are both addressed in detail in the ESI. $\dagger$

\section{Conclusions}

By means of a blend of experiments and simulations, we have unravelled the origins of ice nucleation on cholesterol (CHL), a prototypical organic crystal of relevance to cryopreservation. Our results suggest that its exceptional ice nucleating activity stems from the ability of its flexible hydrophilic surface to form unconventional ice-templating structures - specifically, hydrogen bonded cages comprising 6 - as well as 5 -membered rings. In addition, the experimental evidence reported here suggests that the intrinsic potential of cholesterol to nucleate ice may potentially be enhanced by specific topological features of the crystalline habit. In particular, droplet freezing measurements show that cholesterol promotes the heterogeneous formation of ice across a wide range of temperatures (from -4 to $-20{ }^{\circ} \mathrm{C}$ ). In fact, we find that CHLM crystals nucleate ice far better than the mineral feldspar, which is one of the most effective inorganic ice nucleating agents of relevance to atmospheric science. Moreover, electron microscopy measurements suggest that the broad range of freezing temperatures we observe for CHLM crystals may be due to the coexistence of diverse structural features of the crystalline surface, which in turn can act as different nucleation sites. The microscopic structure of the latter remains to be assessed, but 
the possibility that different parts of the CHLM surface may nucleate ice with different efficiency suggests that surface topography can play an important role in determining the ice nucleating ability of organic crystals.

Surprisingly, we find that CHLM crystals, despite being exceptionally good ice nucleating agents, do not provide a conventional template for ice to form. Specifically, molecular simulations reveal that, as opposed to what has been reported for supercooled water in contact with simple model substrates (e.g. Lennard-Jones crystals, which allow to rapidly explore different surface geometries ${ }^{78}$ ) and/or inorganic materials (such as carbonaceous particles, ${ }^{\mathbf{4 2}}$ or clay minerals $\left.{ }^{\mathbf{4 4 4 5}}\right)$, water on the (001) hydroxylated surface of cholesterol monohydrate (the most abundant interface in aqueous environments) does not form an ordered, dense, ice-like overlayer. Instead, due to the flexibility of the CHLM surface and its relatively low density of hydroxyl groups, water molecules partially infiltrate the crystal, forming a network of both 6- and 5-membered hydrogen bonded rings. The latter involve water molecules as well as hydroxyl groups provided by CHL molecules. While some of these structural features (particularly pentagonal rings) are known to hinder homogeneous water freezing, we find that they actually facilitate the heterogeneous formation of both hexagonal and cubic ice on CHLM crystals. In fact, enhanced sampling simulations suggest the emergence of stacking disordered ice (a mixture of the two polytypes) at the water-CHLM interface. This is in stark contrast with what we have previously observed in the case of $e . g$. the clay mineral kaolinite, where only the hexagonal polytype was observed along the whole nucleation process. ${ }^{44}$ In fact, more often than not a given crystalline substrate nucleates exclusively one of the two ice polytypes. ${ }^{7,13}$ Moreover, we find that the nucleation rate of ice on CHLM crystals is basically identical to that we have previously calculated in the case of kaolinite - at the same strong supercooling $\left(\Delta T_{\mathrm{S}}=42 \mathrm{~K}\right)$. Kaolinite and CHLM are both characterised by an hydrogen bond network capable of facilitating the formation of ice nuclei: thus, the substantial difference in the ice nucleating ability we observe experimentally for these two compounds is most likely rooted into their surface topography. In fact, the $n_{s}$ data reported in Fig. $1 \mathrm{~b}$ suggest that two populations of potentially different ice nucleating sites may coexist on the CHLM surface. The change in the slope of the CHLM data is reminiscent of that observed for freezing spectra for birch pollen, ${ }^{\mathbf{1 7}, \mathbf{1 0 0}}$ which has been attributed to the presence of two different ice nucleating macromolecules. ${ }^{101}$ Similarly, we argue that there may be two different broad classes of ice nucleating sites on CHLM, represented by the two different slopes in the freezing spectra. Due to the spatially sporadic nature of the highly active sites, which are not present in every millimetre diameter droplet, it seems likely that these two different classes of ice nucleation sites are related to specific defects or the diverse topography of the CHLM, rather than any factors related to the bulk molecular structure of CHLM.

In addition, the emergence of stacking disordered ice phases during the heterogeneous formation of ice has been experimentally observed, ${ }^{99}$ and consequently ascribed to different crystal growth regimes. Our results offer the intriguing prospect that the nucleation process itself may favour, in some cases, the formation of stacking disordered ices. Thus, we argue that the dramatic ice nucleation ability of certain organic materials may be traced down not only to the formation of a network of hydrogen bonds between water and the nucleation sites, but also to the capability of specific surfaces to promote at the same time different ice polytypes as a function of supercooling. In order to verify this hypothesis, though, we would need to investigate ice nucleation on CHLM at milder supercooling. To this end, an heterogeneous seeded molecular dynamics approach is currently being validated. ${ }^{\mathbf{1 0 2}}$ Our results also suggest that organic crystals sit in between inorganic and biological materials, when it comes to promoting the formation of ice: substrates like CHLM are characterised by relatively flat surfaces exposing an array of amphoteric functional groups, much like several inorganic ice nucleating agents (e.g. kaolinite, feldspar, hydroxylated graphene), but the flexibility of the surface and the low density of such functional groups is typical of biological nucleating agents such as macromolecules and bacterial fragments. This is especially relevant in the case of CHL, a molecule which is not only used in crystalline form as an ice nucleating agent in cryopreservation applications, but that significantly contributes to the composition of animal cell membranes as well.

In summary, the experiments and simulations presented in this work indicate that cholesterol crystals are incredibly efficient ice nucleating agents, active across a broad range of supercooling. We show that such strong ice nucleating activity is due to the intrinsic potential of the flexible amphoteric surfaces of CHLM to form unconventional ice-templating molecular structures. It is likely that microscopic structural features of the crystals could further enhance the ability of CHLM (and potentially of other organic crystals) to form ice, by offering a diverse array of nucleating sites. In fact, we believe that for an ice nucleating agent to be very efficient, a combination of interfacial "chemistry" and surface topography is generally required. This interplay could thus be the key to understand the heterogeneous formation of ice on molecular organic crystals, and it may provide a starting point for the investigation of ice in soft and biological matter at the molecular level. In particular, tailoring the microscopic structure of the substrate and modifying the nature as well as the density of hydrogen-bonding functional groups at the water-substrate interface can be seen as two different routes to engineer the ice nucleating ability of novel cryoprotectants, the design of which, at the moment, largely relies on the high-throughput screening of whole libraries of different compounds. The absence of a proper structure-to-function paradigm is perhaps the most pressing challenge in cryobiology: this is why future work will be devoted to assess whether and how hydrogen-bonding functional groups other than hydroxyls would be equally effective to enhance the kinetics of heterogeneous ice nucleation.

\section{Conflicts of interest}

There are no conflicts to declare. 


\section{Acknowledgements}

This work was supported by the European Research Council (ERC) under the European Union's Seventh Framework Programme: Grant Agreement number 616121 (HeteroIce project [AM, PP, GCS]), Grant Agreement number 713664 (CryoProtect project [TFW, BJM]) and Grant Agreement number 648661 (MarineIce project [TFW, BJM]). MAH and BJM are also supported by the Engineering Physical Sciences Research Council (EPSRC) via the Research Grant number EP/M003027/1. GCS and PP acknowledge the use of the UCL Grace and Legion High Performance Computing Facilities, the use of Emerald, a GPUaccelerated High Performance Computer, made available by the Science \& Engineering South Consortium operated in partnership with the STFC Rutherford-Appleton Laboratory and the use of ARCHER UK National Supercomputing Service (http:// www.archer.ac.uk) through the Materials Chemistry Consortium via the EPSRC grant number EP/L000202. The Forward Flux Sampling simulations were supported by a grant from the Swiss National Supercomputing Centre (CSCS; project ID s758).

\section{References}

1 T. Bartels-Rausch, Nature, 2013, 494, 27-29.

2 E. Mocé, E. Blanch, C. Tomás and J. Graham, Reprod. Domest. Anim., 2010, 45, 57-66.

3 I. Massie, C. Selden, H. Hodgson, B. Fuller, S. Gibbons and G. J. Morris, Tissue Eng., Part C, 2014, 20, 693-702.

4 J. Morris and E. Acton, Cryobiology, 2013, 66, 85-92.

5 B. J. Murray, D. O'Sullivan, J. D. Atkinson and M. E. Webb, Chem. Soc. Rev., 2012, 41, 6519-6554.

6 B. Slater, A. Michaelides, C. G. Salzmann and U. Lohmann, Bull. Am. Meteorol. Soc., 2015, 97, 1797-1802.

7 G. C. Sosso, J. Chen, S. J. Cox, M. Fitzner, P. Pedevilla, A. Zen and A. Michaelides, Chem. Rev., 2016, 116, 7078-7116.

8 U. Lohmann and J. Feichter, Atmos. Chem. Phys., 2005, 5, 715-737.

9 I. Tan, T. Storelvmo and M. D. Zelinka, Science, 2016, 352, 224-227.

10 C. Marcolli, B. Nagare, A. Welti and U. Lohmann, Atmos. Chem. Phys., 2016, 16, 8915-8937.

11 J. D. Atkinson, B. J. Murray, M. T. Woodhouse, T. F. Whale, K. J. Baustian, K. S. Carslaw, S. Dobbie, D. O'Sullivan and T. L. Malkin, Nature, 2013, 498, 355-358.

12 A. D. Harrison, T. F. Whale, M. A. Carpenter, M. A. Holden, L. Neve, D. O'Sullivan, J. Vergara Temprado and B. J. Murray, Atmos. Chem. Phys., 2016, 16, 10927-10940.

13 A. Kiselev, F. Bachmann, P. Pedevilla, S. J. Cox, A. Michaelides, D. Gerthsen and T. Leisner, Science, 2017, 355, 367-371.

14 L. R. Maki, E. L. Galyan, M.-M. Chang-Chien and D. R. Caldwell, Appl. Microbiol., 1974, 28, 456-459.

15 J. Lindemann, G. J. Warren and T. V. Suslow, Science, 1986, 231, 536.

16 R. Pandey, K. Usui, R. A. Livingstone, S. A. Fischer, J. Pfaendtner, E. H. G. Backus, Y. Nagata, J. FröhlichNowoisky, L. Schmüser, S. Mauri, J. F. Scheel,
D. A. Knopf, U. Pöschl, M. Bonn and T. Weidner, Sci. Adv., 2016, 2, e1501630.

17 B. G. Pummer, H. Bauer, J. Bernardi, S. Bleicher and H. Grothe, Atmos. Chem. Phys., 2012, 12, 2541-2550.

18 M. Bar Dolev, I. Braslavsky and P. L. Davies, Annu. Rev. Biochem., 2016, 85, 515-542.

19 W. Asghar, R. E. Assal, H. Shafiee, R. M. Anchan and U. Demirci, Biotechnol. J., 2014, 9, 895-903.

20 A. Aijaz, M. Li, D. Smith, D. Khong, C. LeBlon, O. S. Fenton, R. M. Olabisi, S. Libutti, J. Tischfield, M. V. Maus, R. Deans, R. N. Barcia, D. G. Anderson, J. Ritz, R. Preti and B. Parekkadan, Nat. Biomed. Eng., 2018, 2, 362-376.

21 N. Fukuta and B. J. Mason, J. Phys. Chem. Solids, 1963, 24, 715-718.

22 N. Fukuta, J. Atmos. Sci., 1966, 23, 191-196.

23 R. B. Head, Nature, 1961, 191, 1058-1059.

24 I. Massie, C. Selden, H. Hodgson and B. Fuller, Tissue Eng., Part C, 2011, 17, 765-774.

25 M. R. Krause and S. L. Regen, Acc. Chem. Res., 2014, 47, 3512-3521.

26 G. R. Edwards and L. F. Evans, J. Atmos. Sci., 1971, 28, 1443-1447.

27 K. A. Pratt, P. J. DeMott, J. R. French, Z. Wang, D. L. Westphal, A. J. Heymsfield, C. H. Twohy, A. J. Prenni and K. A. Prather, Nat. Geosci., 2009, 2, 398-401.

28 C. Hoose, J. E. Kristjánsson and S. M. Burrows, Environ. Res. Lett., 2010, 5, 024009.

29 B. Wang, A. T. Lambe, P. Massoli, T. B. Onasch, P. Davidovits, D. R. Worsnop and D. A. Knopf, J. Geophys. Res., 2012, 117, D16209.

30 P. L. Davies, Trends Biochem. Sci., 2014, 39, 548-555.

31 K. Liu, C. Wang, J. Ma, G. Shi, X. Yao, H. Fang, Y. Song and J. Wang, Proc. Natl. Acad. Sci. U. S. A., 2016, 113, 1473914744.

32 J. M. Campbell, F. C. Meldrum and H. K. Christenson, Proc. Natl. Acad. Sci. U. S. A., 2017, 114, 810-815.

33 S. A. Zielke, A. K. Bertram and G. N. Patey, J. Phys. Chem. B, 2016, 120, 1726-1734.

34 X.-X. Zhang, M. Chen and M. Fu, J. Chem. Phys., 2014, 141, 124709.

35 Y. Bi, R. Cabriolu and T. Li, J. Phys. Chem. C, 2016, 120, 1507-1514.

36 A. Reinhardt and J. P. K. Doye, J. Chem. Phys., 2014, 141, 084501. 37 G. Fraux and J. P. K. Doye, J. Chem. Phys., 2014, 141, 216101.

38 P. Pedevilla, M. Fitzner and A. Michaelides, Phys. Rev. B, 2017, 96, 115441.

39 L. Lupi, R. Hanscam, Y. Qiu and V. Molinero, J. Phys. Chem. Lett., 2017, 8, 4201-4205.

40 Y. Bi, B. Cao and T. Li, Nat. Commun., 2017, 8, 15372.

41 V. Molinero and E. B. Moore, J. Phys. Chem. B, 2009, 113, 4008-4016.

42 L. Lupi, A. Hudait and V. Molinero, J. Am. Chem. Soc., 2014, 136, 3156-3164.

43 Y. Qiu, N. Odendahl, A. Hudait, R. Mason, A. K. Bertram, F. Paesani, P. J. DeMott and V. Molinero, J. Am. Chem. Soc., 2017, 139, 3052-3064.

44 G. C. Sosso, T. Li, D. Donadio, G. A. Tribello and A. Michaelides, J. Phys. Chem. Lett., 2016, 7, 2350-2355. 
45 G. C. Sosso, G. A. Tribello, A. Zen, P. Pedevilla and A. Michaelides, J. Chem. Phys., 2016, 145, 211927.

46 L. F. Evans, J. Atmos. Sci., 1966, 23, 751-752.

47 M. Gavish, J. L. Wang, M. Eisenstein, M. Lahav and L. Leiserowitz, Science, 1992, 256, 815-818.

48 P. K. Wolber, C. A. Deininger, M. W. Southworth, J. Vandekerckhove, M. v. Montagu and G. J. Warren, Proc. Natl. Acad. Sci. U. S. A., 1986, 83, 7256-7260.

49 D. Gurian-Sherman and S. E. Lindow, FASEB J., 1993, 7, 1338-1343.

50 T. F. Whale, B. J. Murray, D. O'Sullivan, T. W. Wilson, N. S. Umo, K. J. Baustian, J. D. Atkinson, D. A. Workneh and G. J. Morris, Atmos. Meas. Tech., 2015, 8, 2437-2447.

51 C. A. Schneider, W. S. Rasband and K. W. Eliceiri, Nat. Methods, 2012, 9, 671-675.

52 B. M. Craven, Nature, 1976, 260, 727-729.

53 J. L. F. Abascal, E. Sanz, R. G. Fernández and C. Vega, J. Chem. Phys., 2005, 122, 234511.

54 R. S. Abendan and J. A. Swift, Langmuir, 2002, 18, 4847-4853. 55 P. Bjelkmar, P. Larsson, M. A. Cuendet, B. Hess and E. Lindahl, J. Chem. Theory Comput., 2010, 6, 459-466.

56 J. B. Lim, B. Rogaski and J. B. Klauda, J. Phys. Chem. B, 2012, 116, 203-210.

57 A. Haji-Akbari and P. G. Debenedetti, Proc. Natl. Acad. Sci. U. S. A., 2015, 112, 10582-10588.

58 H. A. Lorentz, Ann. Phys., 1881, 248, 127-136.

59 G. Bussi, D. Donadio and M. Parrinello, J. Chem. Phys., 2007, 126, 014101.

60 S. Miyamoto and P. A. Kollman, J. Comput. Chem., 1992, 13, 952-962.

61 B. Hess, H. Bekker, H. J. C. Berendsen and J. G. E. M. Fraaije, J. Comput. Chem., 1997, 18, 1463-1472.

62 R. J. Herbert, B. J. Murray, T. F. Whale, S. J. Dobbie and J. D. Atkinson, Atmos. Chem. Phys., 2014, 14, 8501-8520.

63 T. F. Whale, M. Rosillo-Lopez, B. J. Murray and C. G. Salzmann, J. Phys. Chem. Lett., 2015, 6, 3012-3016.

64 H. S. Shieh, L. G. Hoard and C. E. Nordman, Nature, 1977, 267, 287-289.

65 C. R. Loomis, G. G. Shipley and D. M. Small, J. Lipid Res., 1979, 20, 525-535.

66 N. Garti, L. Karpuj and S. Sarig, J. Lipid Res., 1981, 22, 785791.

67 H. Rapaport, I. Kuzmenko, S. Lafont, K. Kjaer, P. B. Howes, J. Als-Nielsen, M. Lahav and L. Leiserowitz, Biophys. J., 2001, 81, 2729-2736.

68 I. Solomonov, M. J. Weygand, K. Kjaer, H. Rapaport and L. Leiserowitz, Biophys. J., 2005, 88, 1809-1817.

69 R. B. Head, J. Phys. Chem. Solids, 1962, 23, 1371-1378.

70 G. Vali, P. J. DeMott, O. Möhler and T. F. Whale, Atmos. Chem. Phys., 2015, 15, 10263-10270.

71 G. Vali, Atmos. Chem. Phys., 2014, 14, 5271-5294.

$72 \mathrm{H}$. Pruppacher and J. Klett, Microphysics of clouds and precipitation, 1997.

73 G. Vali, Atmos. Chem. Phys., 2008, 8, 5017-5031.

74 C. Gurganus, J. Charnawskas, A. Kostinski and R. Shaw, Phys. Rev. Lett., 2014, 113, 235701.
75 T. F. Whale, M. A. Holden, A. N. Kulak, Y.-Y. Kim, F. C. Meldrum, H. K. Christenson and B. J. Murray, Phys. Chem. Chem. Phys., 2017, 19, 31186-31193.

76 D. Turnbull and B. Vonnegut, Ind. Eng. Chem., 1952, 44, 1292-1298.

77 M. F. Harrach and B. Drossel, J. Chem. Phys., 2014, 140, 174501.

78 M. Fitzner, G. C. Sosso, S. J. Cox and A. Michaelides, J. Am. Chem. Soc., 2015, 137, 13658-13669.

79 H. Shintani and H. Tanaka, Nat. Phys., 2006, 2, 200-206.

80 P. Pedevilla, S. J. Cox, B. Slater and A. Michaelides, J. Phys. Chem. C, 2016, 120, 6704-6713.

81 A. Hudait, N. Odendahl, Y. Qiu, F. Paesani and V. Molinero, J. Am. Chem. Soc., 2018, 140, 4905-4912.

82 R. J. Allen, C. Valeriani and P. Rein ten Wolde, J. Phys.: Condens. Matter, 2009, 21, 463102.

83 T. Li, D. Donadio, G. Russo and G. Galli, Phys. Chem. Chem. Phys., 2011, 13, 19807-19813.

84 T. Li, D. Donadio and G. Galli, Nat. Commun., 2013, 4, 1887. 85 C. Valeriani, E. Sanz and D. Frenkel, J. Chem. Phys., 2005, 122, 194501.

86 Z.-J. Wang, C. Valeriani and D. Frenkel, J. Phys. Chem. B, 2009, 113, 3776-3784.

87 Y. Bi and T. Li, J. Phys. Chem. B, 2014, 118, 13324-13332.

88 M. M. Gianetti, A. Haji-Akbari, M. P. Longinotti and P. G. Debenedetti, Phys. Chem. Chem. Phys., 2016, 18, 4102-4111.

89 A. Haji-Akbari and P. G. Debenedetti, Proc. Natl. Acad. Sci. U. S. A., 2017, 114, 3316-3321.

90 A. Laio and M. Parrinello, Proc. Natl. Acad. Sci. U. S. A., 2002, 99, 12562-12566.

91 P. G. Bolhuis, D. Chandler, C. Dellago and P. L. Geissler, Annu. Rev. Phys. Chem., 2002, 53, 291-318.

92 J. R. Espinosa, C. Vega, C. Valeriani and E. Sanz, J. Chem. Phys., 2016, 144, 034501.

93 A. Haji-Akbari, J. Chem. Phys., 2018, 149, 072303.

94 G. A. Tribello, F. Giberti, G. C. Sosso, M. Salvalaglio and M. Parrinello, J. Chem. Theory Comput., 2017, 13, 1317-1327.

95 M. Fitzner, G. C. Sosso, F. Pietrucci, S. Pipolo and A. Michaelides, Nat. Commun., 2017, 8, 2257.

96 R. Cabriolu and T. Li, Phys. Rev. E, 2015, 91, 052402.

97 E. B. Moore and V. Molinero, Phys. Chem. Chem. Phys., 2011, 13, 20008.

98 T. C. Hansen, M. M. Koza, P. Lindner and W. F. Kuhs, J. Phys.: Condens. Matter, 2008, 20, 285105.

99 T. L. Malkin, B. J. Murray, C. G. Salzmann, V. Molinero, S. J. Pickering and T. F. Whale, Phys. Chem. Chem. Phys., 2014, 17, 60-76.

100 D. O'Sullivan, B. J. Murray, J. F. Ross, T. F. Whale, H. C. Price, J. D. Atkinson, N. S. Umo and M. E. Webb, Sci. Rep., 2015, 5, 8082.

101 S. Augustin, H. Wex, D. Niedermeier, B. Pummer, H. Grothe, S. Hartmann, L. Tomsche, T. Clauss, J. Voigtlâd'nder, K. Ignatius and F. Stratmann, Atmos. Chem. Phys., 2013, 13, 10989-11003.

102 P. Pedevilla, M. Fitzner, G. C. Sosso and A. Michaelides, J. Chem. Phys., 2018, 072327. 